\title{
Electron sharing and localization in real space for the Mott transition from 1RDMFT periodic calculations
}

\author{
Alexey I. Baranov ${ }^{1}$ • Ángel Martín Pendás ${ }^{2}$
}

Received: 15 March 2017 / Accepted: 31 July 2017 / Published online: 9 August 2017

(C) The Author(s) 2017. This article is an open access publication

\begin{abstract}
One-particle reduced density matrix functional theory (1RDMFT) has been applied for the investigation of the real-space picture of the Mott transitions in archetypal hydrogen lattices. The evolution of the degree of electron localization during the transition has been evaluated using electron sharing indices for QTAIM atoms, and the connection between the strength of electronic correlation expressed by the 1RDMFT correlation energy and the degree of electron localization has been analyzed at various stages of transition. The spatial decay of the electron sharing indices in the course of transition has also been evaluated and compared with the results obtained for the Hubbard model and finite cluster calculations.
\end{abstract}

Keywords Electron sharing indices · Mott transition · Reduced density matrix functional theory

\section{Introduction}

Mott insulators and the closely related Mott metal-insulator transitions play an important role [1-3] in the modern theory

Authors thank Deutsche Forschungsgemeinschaft for provided computational resources within the Project BA-4911/1-1.

Published as part of the special collection of articles "First European Symposium on Chemical Bonding".

Alexey I. Baranov

baranov@cpfs.mpg.de

1 Max-Planck-Institute for Chemical Physics of Solids, Nöthnitzer Str. 40, 01187 Dresden, Germany

2 Departmento de Química Física y Analítica, Universidad de Oviedo, C/ Julián Clavería 8, 33006 Oviedo, Spain of the electronic structure of matter. The most important family of such compounds is found for systems containing d- and f-electrons, many of which are known to possess fascinating properties, for instance, high-temperature superconductivity or colossal magnetoresistance. After many decades of intensive investigations, Mott insulators still stay in the focus of active research, ${ }^{1}$ being especially difficult objects to deal with from the theoretical viewpoint. The reason behind that difficulty is that they are essentially governed by strong electron correlation which makes them complicated interacting many-body systems for which one-electron theories often turn out to be even qualitatively wrong [4].

Due to this complexity, lots of efforts have been put on the study of prototypical systems: from analytical models, e.g., Hubbard models [5, 6], allowing even exact solutions under certain circumstances [7] to hydrogen lattices handled by a number of advanced numerical techniques like dynamic mean-field theory (DMFT), [8], density matrix embedding theory (DMET) $[9,10]$ variational Quantum Monte-Carlo (QMC) [11] and others. The first nonempirical theoretical descriptions of Mott transitions in 3D metal solid-state compounds employing, e.g., DMFT [12] or one-electron reduced density matrix functional theory (1RDMFT) [13] have also appeared recently.

Mott transitions (MTs) are tightly related to the paradigmatic homolytic dissociation of covalent bonds in molecules [14]. Finite clusters from hydrogen lattices have been therefore also extensively used as model systems for studying the MT, with a wide variety of methods ranging from full CI, used ubiquitously as a reference, to density matrix renormalization group [15], auxiliary-field QMC

\footnotetext{
${ }_{1}$ Rev. Mod. Phys. published in 1998 an extensive review under the same title [3] as one written by Mott [1] 30 years before.
} 
[16], variational two-particle reduced density matrix functional theory [17], geminal-based AP1roG methods [18] and many others.

The rationalization of Mott insulators and MTs is not a trivial task since a lot of concepts in both physics and chemistry rely upon one-particle pictures that become inapplicable in this case. Besides the evolution of the total energy, various indicators from spectral densities [8] to the off-diagonal elements of the one-particle reduced density matrix (1-RDM) [17] have been employed to monitor the transition from the metal to the insulator. From this side, it would be advantageous to utilize a universal set of indicators capable to deliver physically sound information from all the models and theoretical approaches. The methods of quantum chemical topology (QCT) [19] can serve as such a universal toolkit for recovering chemical concepts. Being based on orbital invariant reduced density matrices [20], they have been already successfully applied to the study of dissociation processes in simple molecules [21], MTs in model 1D Hubbard lattices and finite hydrogen cyclic molecules [22].

This article presents the application of this powerful toolkit to the study of the Mott transition in one- to threedimensional hydrogen lattices evaluated from the results of periodic one-particle reduced density matrix functional theory (1RDMFT) calculations [23] employing APW+lo+LO basis set [24]. The degree of electron localization and electron sharing in real space has been studied employing electron sharing indices [25, 26], and the evolution of their profiles during the MT is analyzed as well as their spatial dependence at the different stages of the transition. The influence of the electronic correlation on the electron localization and sharing during the MT is also considered. Finally, the dependence of the results from the computational parameters has been also analyzed.

\section{Methods and computational details}

\subsection{One-electron reduced density matrix functional theory}

In 1RDMFT the system can be described by its one-electron reduced density matrix (1RDM) [20]

$\gamma\left(\mathbf{x}, \mathbf{x}^{\prime}\right)=N \int d \mathbf{x}_{2} \ldots \int d \mathbf{x}_{N} \Psi^{*}\left(\mathbf{x}, \mathbf{x}_{2} \ldots \mathbf{x}_{N}\right) \Psi\left(\mathbf{x}^{\prime}, \mathbf{x}_{2} \ldots \mathbf{x}_{N}\right)$,

where $\Psi$ is the $N$-electron wavefunction of the system and $\mathbf{x}=\{\mathbf{r}, \sigma\}$ denotes a combined space and spin electron coordinate. In the electronic energy [23] functional of 1RDM theory,

$$
\begin{aligned}
E[\gamma]= & -\frac{1}{2} \int d \mathbf{x} \int d \mathbf{x}^{\prime} \delta\left(\mathbf{x}-\mathbf{x}^{\prime}\right) \nabla_{\mathbf{x}}^{2} \gamma\left(\mathbf{x}, \mathbf{x}^{\prime}\right) \\
& +\int d \mathbf{x} V(\mathbf{x}) \gamma(\mathbf{x}, \mathbf{x}) \\
& +\frac{1}{2} \int d \mathbf{x} \int d \mathbf{x}^{\prime} \frac{\gamma(\mathbf{x}, \mathbf{x}) \gamma\left(\mathbf{x}^{\prime}, \mathbf{x}^{\prime}\right)}{\left|\mathbf{r}-\mathbf{r}^{\prime}\right|} \\
& -\frac{1}{2} \int d \mathbf{x} \int d \mathbf{x}^{\prime} \frac{\gamma\left(\mathbf{x}, \mathbf{x}^{\prime}\right) \gamma\left(\mathbf{x}^{\prime}, \mathbf{x}\right)}{\left|\mathbf{r}-\mathbf{r}^{\prime}\right|}+E_{c}[\gamma]
\end{aligned}
$$

all the terms but the last one, corresponding to the correlation energy, are known exactly. The spin-summed diagonal part of $\gamma$ is the electron density, $\rho(\mathbf{r})=\sum_{\sigma} \gamma(\mathbf{r} \sigma, \mathbf{r} \sigma)$. The $1 \mathrm{RDM}$ for collinear periodic system can be represented via its eigenvalues $\{n\}$ and eigenvectors $\{\psi\}$ called natural occupations and natural spinorbitals, respectively, as:

$\gamma\left(\mathbf{x}, \mathbf{x}^{\prime}\right)=\sum_{j \mathbf{k}} n_{j \mathbf{k} \sigma} \psi_{j \mathbf{k}}^{*}(\mathbf{r}) \psi_{j \mathbf{k}}\left(\mathbf{r}^{\prime}\right) \delta_{\sigma, \sigma^{\prime}}$,

whereas in periodic systems natural spinorbitals have to be Bloch states due to translational symmetry [27]. The N-representability conditions [28] that define appropriate 1-RDMs for a fermionic system (corresponding to either pure state or an ensemble of pure states) are known to be:

$\sum_{j \mathbf{k} \sigma} n_{j \mathbf{k} \sigma}=N, \quad 0 \leq n_{j \mathbf{k}} \leq 1$.

Minimization of Eq. 2 with respect to natural occupations and orbitals yields the best possible 1RDM [23] if we keep the conditions of Eq. 4 fulfilled and the natural orbitals orthonormal. Unfortunately, the exact correlation functional $E_{c}[\gamma]$ is not known, so many approximations to it, starting from Müller's functional [29], have been proposed in the literature [30]. New functionals continue to appear [27, 31, 32].

In the present work a power functional $[27,33]$

$E_{c}[\gamma]=E[\{n\},\{\psi\}]=\frac{1}{2} \sum_{j j^{\prime} \mathbf{k} \mathbf{k}^{\prime} \sigma}\left[n_{j \mathbf{k} \sigma} n_{j^{\prime} \mathbf{k}^{\prime} \sigma}-\left(n_{j \mathbf{k} \sigma} n_{j^{\prime} \mathbf{k}^{\prime} \sigma}\right)^{\lambda}\right] K_{j \mathbf{k} j^{\prime} \mathbf{k}^{\prime}}$

with $\lambda=0.656$ and $K_{j \mathbf{k}^{\prime} \mathbf{k}^{\prime}}=\left\langle\psi_{j \mathbf{k}}(1) \psi_{j^{\prime} \mathbf{k}^{\prime}}(2)\left|r_{12}^{-1}\right| \psi_{j^{\prime} \mathbf{k}^{\prime}}(1) \psi_{j \mathbf{k}}(2)\right\rangle$ has been used because it is known to reproduce the basic features of the electronic structures of some transition metal oxide Mott insulators [13]. Moreover, a benchmarking of the 1RDMFT functionals versus the exact solutions for the quasi-solvable few-electron harmonium atoms [34] yielded for the strongly correlated regime optimal $\lambda$ values reasonably close to the one used here.

1RDMFT calculations have been performed for 1D, 2D (square) and 3D (primitive cubic) hydrogen lattices using a customized version of the Elk code [35]. In the case of 1D and 2D lattices, the distance between lattice replicas was set equal to 10 a.u. The unit cell parameter has been varied within the 
range 2-10 a.u, and the size of the k-mesh along each direction of the lattice was set equal to 16 for $1 \mathrm{D}$ and $2 \mathrm{D}$ calculations and to 8 for the $3 \mathrm{D}$ case.

Natural orbitals have been expanded over the set of Kohn-Sham orbitals (within the local density approximation (LDA) [36]) for the seven lowest bands obtained using periodic APW+lo+LO calculations with the same computational parameters. In a first step, their coefficients have been minimized using fixed step iterative gradient optimization with the consequent orthogonalization until the energy change between the two last iterations becomes less than $10^{-5}$ a.u. In a second step, the natural occupations have been minimized using fixed step iterative gradient optimization until the energy change becomes less than $10^{-8}$ a.u. These sequence has been repeated at least three times which has provided a final convergence in the total electronic energy at the level $<1 \times 10^{-4}$ a.u.

To check the sensitivity of the results, extra 1RDMFT calculations employing:

- 10 lowest bands instead of 7 for 1D lattices,

- 24 k-divisions instead of 16 for 1D lattices,

- $12 \mathrm{k}$-divisions instead of 8 for 3D lattices, and

- Müller's functional [29] with $\lambda=0.5$ instead of the power functional already described [13] functional for 1D lattices

have been performed and evaluated.

\subsection{APW+lo+LO method}

Augmented planewaves with local orbitals belong to one of the most accurate and popular classes of basis sets for all electron full-potential calculations [24, 37]. A single APW oneelectron function is a multipole-expanded function within the atom-centered nonoverlapping muffin-tin (MT) spheres and a planewave outside, in the interstitial region (IR):

$\phi_{n \mathbf{k}+\mathbf{G}}(\mathbf{r})= \begin{cases}\sum_{l m} f_{n, l m}^{\mathbf{k}+\mathbf{G}}(r) Y_{l m}(\hat{\mathbf{r}}) & \mathbf{r} \in \mathrm{MT} \\ \frac{1}{\sqrt{\Omega}} e^{i(\mathbf{k}+\mathbf{G}) \mathbf{r}} & \mathbf{r} \in \mathrm{IR} .\end{cases}$

The radial functions $f_{n, l m}^{\mathbf{k}+\mathbf{G}}(r)$ are built from the solutions of radial Schrödinger equations taken at fixed energy values. In the APW+lo method, APWs are extended with several additional so-called local functions (defined only inside the MT) that are used to improve the representation of radial parts. With this, the Kohn-Sham orbitals can then be expanded in the APW basis as:

$\psi_{j \mathbf{k}}(\mathbf{r})=\sum_{\mathbf{G}} \sum_{n} c_{j ; n \mathbf{k}+\mathbf{G}} \phi_{n \mathbf{k}+\mathbf{G}}(\mathbf{r})$.

\subsection{Electron sharing indices}

The degree of electron localization inside the spatial domain $\Omega$, given by the localization index (LI) $\lambda(\Omega)$ [25], can be evaluated as

$$
\begin{aligned}
\lambda(\Omega) & =\int_{\Omega} d \mathbf{r}_{1} \int_{\Omega} d \mathbf{r}_{2}\left[\rho\left(\mathbf{r}_{1}\right) \rho\left(\mathbf{r}_{2}\right)-\rho_{2}\left(\mathbf{r}_{1}, \mathbf{r}_{2}\right)\right] \\
& =\int_{\Omega} d \mathbf{r}_{1} \int_{\Omega} d \mathbf{r}_{2} \rho_{x c}\left(\mathbf{r}_{1}, \mathbf{r}_{2}\right),
\end{aligned}
$$

where $\rho_{2}\left(\mathbf{r}_{1}, \mathbf{r}_{2}\right)$ is the electron pair density,

$$
\begin{aligned}
\rho_{2}\left(\mathbf{r}_{1}, \mathbf{r}_{2}\right)= & N(N-1) \int d \mathbf{r}_{3} \ldots \\
& \int d \mathbf{r}_{N} \Psi^{*}\left(\mathbf{r}_{1}, \mathbf{r}_{2} \ldots \mathbf{r}_{N}\right) \Psi\left(\mathbf{r}_{1}, \mathbf{r}_{2} \ldots \mathbf{r}_{N}\right),
\end{aligned}
$$

and the expression in the square brackets is the so-called exchange-correlation part of the electron pair density [38].

The difference between the (average) domain population $N(\Omega)=\int_{\Omega} d \mathbf{r} \rho(\mathbf{r})$ and the localization index

$N(\Omega)-\lambda(\Omega)=\sigma^{2}(\Omega)$

is equal to the statistical variance of the domain population [25]. If $\lambda(\Omega)$ is equal to the electron population of domain $\Omega$, then the electrons are regarded as perfectly localized inside it.

Delocalization indices (DI) $\delta\left(\Omega, \Omega^{\prime}\right)$ characterize the degree of electron delocalization between the corresponding spatial domains and are given by

$$
\begin{aligned}
\delta\left(\Omega, \Omega^{\prime}\right) & =2 \int_{\Omega} d \mathbf{r}_{1} \int_{\Omega^{\prime}} d \mathbf{r}_{2}\left[\rho\left(\mathbf{r}_{1}\right) \rho\left(\mathbf{r}_{2}\right)-\rho_{2}\left(\mathbf{r}_{1}, \mathbf{r}_{2}\right)\right] \\
& =2 \int_{\Omega} d \mathbf{r}_{1} \int_{\Omega^{\prime}} d \mathbf{r}_{2} \rho_{x c}\left(\mathbf{r}_{1}, \mathbf{r}_{2}\right) .
\end{aligned}
$$

For spatial domains taken to be QTAIM topological basins [39], they can be interpreted as a covalent bond order between QTAIM atoms [40, 41].

The sum of delocalization indices between all neighboring domains $\Omega^{\prime}$ surrounding one particular $\Omega$ is directly related to the statistical variance of the domain population

$2 \sigma^{2}(\Omega)=\sum_{\Omega^{\prime} \neq \Omega} \delta\left(\Omega, \Omega^{\prime}\right)$

which can be interpreted as the valence of the QTAIM atom $\Omega$ [41-43].

Equations 8 and 11 require the pair or exchange-correlation (xc) hole density to be accessible. However, no exact expressions for them via the RDM are known, so we have evaluated the pair density via an approximated expression of the xc-hole density. In this work, we have used the form proposed by Buijse and Baerends [44] 
Table 1 Comparison of localization and delocalization indices for hydrogen lattices from DFT [47] and the present 1RDMFT calculations

\begin{tabular}{lllll}
\hline & Data from & ${ }_{\infty}^{1}[\mathrm{H}]$ & ${ }_{\infty}^{2}[\mathrm{H}]$ & ${ }_{\infty}^{3}[\mathrm{H}]$ \\
\hline$\delta\left(\mathrm{H}, \mathrm{H}^{\prime}\right)$ & 1RDMFT & 0.384 & 0.19 & 0.12 \\
$\delta\left(\mathrm{H}, \mathrm{H}^{\prime}\right)$ & DFT & 0.424 & 0.19 & 0.12 \\
$\lambda(\mathrm{H})$ & 1RDMFT & 0.59 & 0.49 & 0.43 \\
$\lambda(\mathrm{H})$ & DFT & 0.46 & 0.37 & 0.31 \\
\hline
\end{tabular}

$\lambda(\mathrm{H})$ - localization index for $\mathrm{H} ; \delta\left(\mathrm{H}, \mathrm{H}^{\prime}\right)$ - delocalization index between nearest $\mathrm{H}$ atoms $\left(d\left(\mathrm{H}, \mathrm{H}^{\prime}\right)=2.5\right.$ a.u. $)$. All the values are obtained using Fulton's formulation [45]

$\rho_{x c}\left(\mathbf{r}_{1}, \mathbf{r}_{2}\right)=\sum_{j j^{\prime} \mathbf{k} \mathbf{k}^{\prime}}\left(n_{j \mathbf{k}} n_{j^{\prime} \mathbf{k}^{\prime}}\right)^{1 / 2} \psi_{j \mathbf{k}}^{*}\left(\mathbf{r}_{1}\right) \psi_{j^{\prime} \mathbf{k}^{\prime}}^{*}\left(\mathbf{r}_{2}\right) \psi_{j \mathbf{k}}\left(\mathbf{r}_{2}\right) \psi_{j^{\prime} \mathbf{k}^{\prime}}\left(\mathbf{r}_{1}\right)$

(representing a Müller-type functional [29], also of power type), since it guarantees the proper normalization of the pair density to $N(N-1)$ pairs which is very convenient for chemical bonding analyses. In this way, computed localization and delocalization indices correspond to the Fulton formulation [45]. All the electron sharing indices as well as the QTAIM partitionings have been calculated using a customized version of DGrid code [46].

\section{Results and discussion}

We will first report and discuss the results of 1RDMFT calculations with $\lambda=0.656$ for $1 \mathrm{D}-3 \mathrm{D}$ hydrogen lattices, comparing them with those from other methods and models reported in the literature. The evolution of electron localization and sharing in the course of the MT, as well as the spatial decay of electron sharing at different stages of transition, will be analyzed in detail, as well as how they are linked to the electronic correlation energy of 1RDMFT (Eq. 5). Finally, we will discuss the stability of the results with respect to the variation of the computational parameters.

Table 1 compares the values of delocalization indices and related parameters obtained from LDA DFT [47] and 1RDMFT calculations for 1D-3D lattices with an small unit cell parameter $a=2.5$ a.u. corresponding to the metallic regime. The nearest neighbor delocalization indices are pretty close for both methods, indicating much similarity in the degree of nearest neighbor sharing. At the same time, the localization indices are systematically higher for 1RDMFT results which thus favors a more localized picture and weaker sharing with distant atoms. This difference can be attributed to the enhanced long-range electron correlation characteristic of the 1RDMFT approximation that favors electron localization [48].

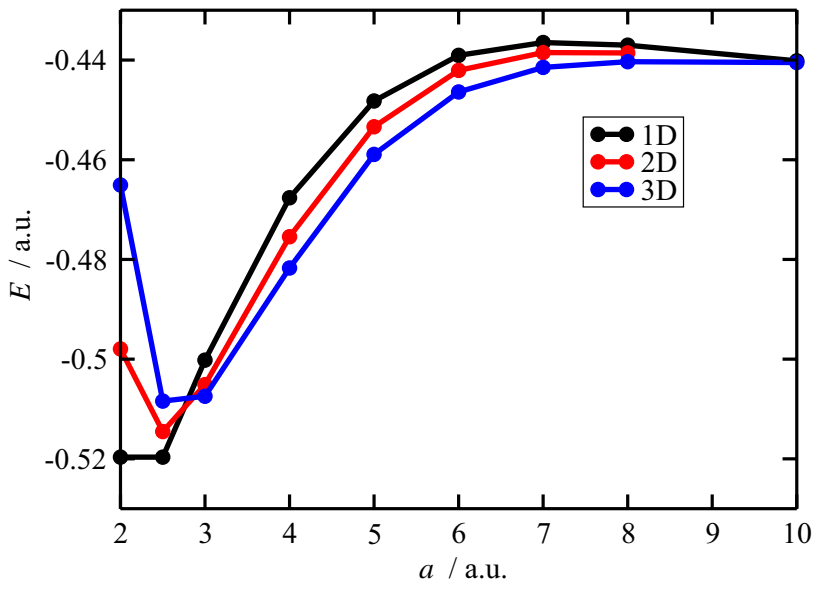

Fig. 1 Dependence of the total energy per atom on the cell parameter for 1D-3D hydrogen lattices from 1RDMFT calculations

Figure 1 presents the dependence of the total energy per atom of hydrogen lattices versus the lattice unit cell parameter, $a$. For $1 \mathrm{D}$ and 2D lattices, the energy profiles display a shallow maximum for large $a$ values which most likely should be attributed to spurious interaction effects among the periodic replicas of the lattices. The energy of dissociated lattices (at 10 a.u.) for all three lattices is very close and roughly approaches -0.44 a.u. which is 0.06 a.u. higher than the exact value. Equilibrium unit cell parameters for all the three lattices lie between 2 and 3 a.u., whereas $a_{1 \mathrm{D}}^{\mathrm{eq}}<a_{2 \mathrm{D}}^{\mathrm{eq}}<a_{3 \mathrm{D}}^{\mathrm{eq}}$. Cohesive energies lie within the range 0.06-0.08 a.u. and decrease in the series $E_{1 \mathrm{D}}^{\mathrm{coh}}>E_{2 \mathrm{D}}^{\mathrm{coh}}>E_{3 \mathrm{D}}^{\mathrm{coh}}$.

Unit cell parameters and cohesive energies coincide reasonably well with the results of other calculations. For finite cluster models of 1D lattices, density matrix renormalization group (DMRG) calculations yielded [15] $a^{\text {eq }} \approx 1.8$ a.u. and $E^{\text {coh }} \approx 0.07$ a.u., similar to AP1 roG [18], QMC [16] and DMET [10] results. For 2D cluster model, the latter method yielded $a^{\text {eq }} \approx 2.5$ a.u. and $E^{\text {coh }} \approx 0.02$ a.u [10]. Variational two-particle reduced density matrix functional theory [17] yielded for finite cluster model of $3 \mathrm{D}$ lattice equilibrium lattice constant is $a^{\mathrm{eq}} \approx 2.8 \mathrm{a}$.u. and $E^{\text {coh }} \approx 0.02$ a.u. Equilibrium unit cell parameters have pretty close values, whereas the cohesive energies show larger deviations most likely due to the difference in the models (finite vs. periodic). It should be noted that the value of $\lambda$ may essentially influence the quality of the potential energy surface [49].

Figure 2 shows the distribution of the occupation numbers of the natural orbitals on $a$ for the 1D lattices. At short distances most of the orbitals are either doubly occupied or empty, and at long distances all of them become singly occupied, a limit which corresponds to the set of noninteracting $\mathrm{H}$ atoms. Higher-dimensional systems show 


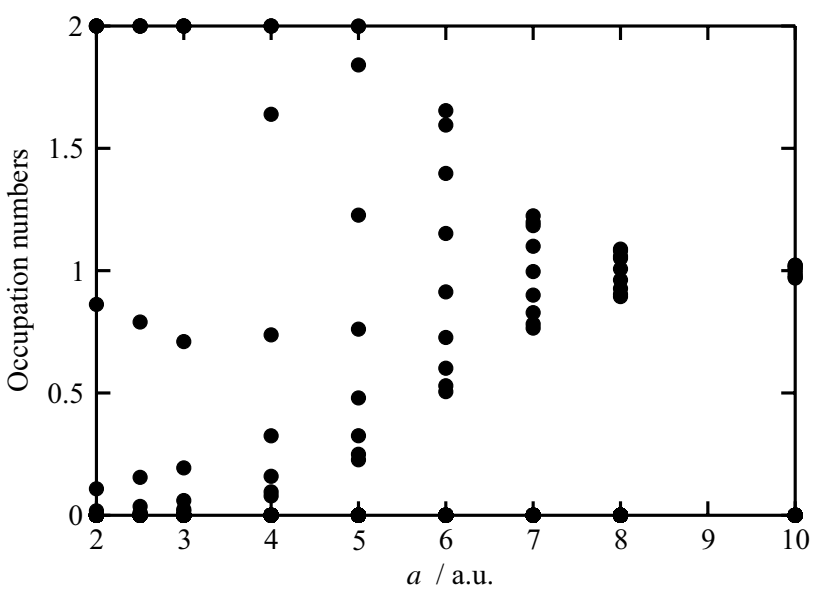

Fig. 2 Distribution of the occupation numbers of the natural orbitals for 1D hydrogen lattice as obtained from 1RDMFT calculations

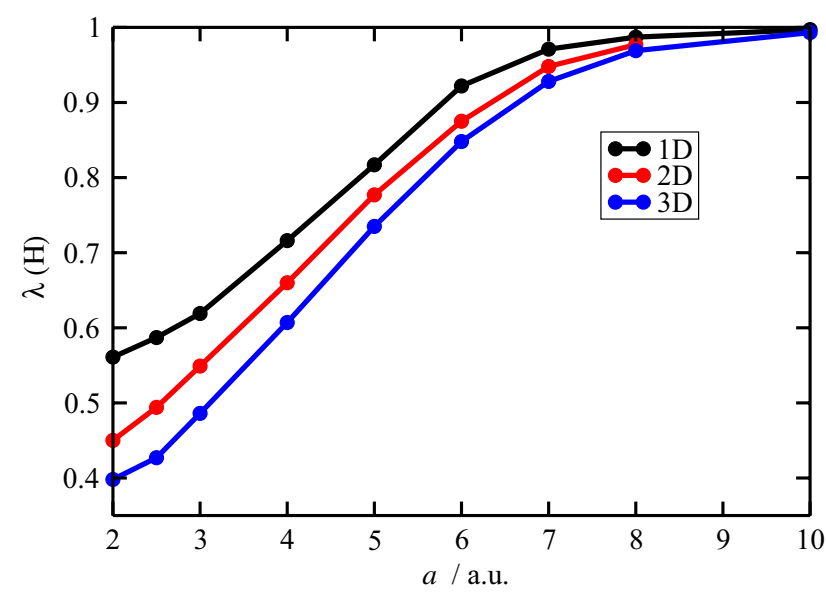

Fig. 3 Dependence of the localization index for QTAIM atoms, $\lambda(H)$, on the cell parameter $a$ for hydrogen lattices in 1RDMFT calculations

similar distributions displaying slightly large dispersion that increases with the dimensionality of the system. A similar evolution of the occupation numbers has been reported from variational 2RDMFT calculations [17].

The behavior of the localization indices of the lattices as $a$ is varied is presented in Fig. 3. In contrast to the local spin density approximation (LSDA) results [50], where the electron localization was shown to display a stepwise increase necessarily associated with the appearance of nonzero magnetic moment, the 1RDMFT results show an electron localization profile that continuously increases from 0.5 to 1 while the system stays nonmagnetic. It is also noteworthy to recognize that the different dimensionality profiles never cross within the distance range studied covering the metal to insulator transition. Thus, the degree of electron localization always decreases as the dimensionality of the system increases. The same effect has been

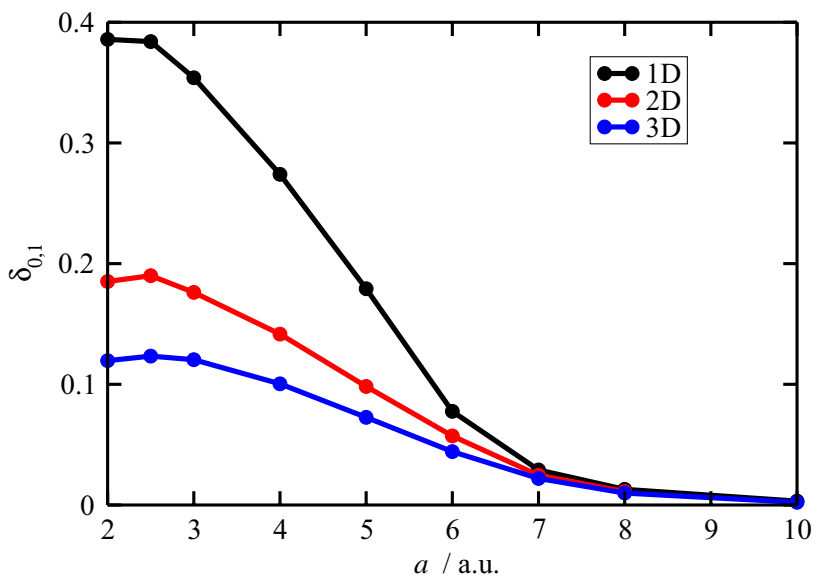

Fig. 4 Dependence of the delocalization index between nearest QTAIM atoms $\delta_{0,1}$ on the cell parameter $a$ for hydrogen lattices in 1RDMFT calculation

observed earlier for lattices in the metallic regime using DFT calculations $[47,51]$ and has been rationalized as a systematic increase of electron sharing due to increase of coordination number in higher-dimensional lattices.

The evolution of the delocalization indices between nearest neighbor QTAIM atoms is shown in Fig. 4. One can clearly recognize smooth sigmoidal profiles much similar to what was reported for finite systems [21] and 1D Hubbard models [22]. We interpret these results in terms of an essential electron sharing between nearest atoms in the metallic regime, just like between covalently bonded atoms in textbook molecules, that gradually decreases to zero in the insulating state during the course of the MT. As it was stated for localization indices, the nearest neighbor delocalization indices also decrease with the system dimensionality for all the distance range studied.

All the nearest neighbor profiles have a sigmoidal shape. From the analysis of molecular systems, it has been stated [21] that such a shape is a sign of shared interactions of the atom with its environment, just as expected for metallic systems. Similar profiles have been obtained from the 1D Hubbard model and in correlated molecular calculations [22].

From the analysis of delocalization indices profiles in molecules [21], it has also been concluded that the inflection point in these profiles signals the situation where bonds are half-broken. Similarly, the analysis of Hubbard model results [22] has showed that at this point the strength of the correlation and hopping terms become equal. The delocalization index value attains at this point half of its maximum value (i.e., at the equilibrium geometry). This rule is also fulfilled for the profiles shown in Fig. 4. With that idea, the middle point of the transition for each lattice can be estimated. One can easily see that this occurs at $\approx 5$ a.u. for the $1 \mathrm{D}$ lattice, at $\approx 5.5$ a.u. for the $2 \mathrm{D}$ case, and at $\approx 6$ a.u. for the $3 \mathrm{D}$ lattice. 


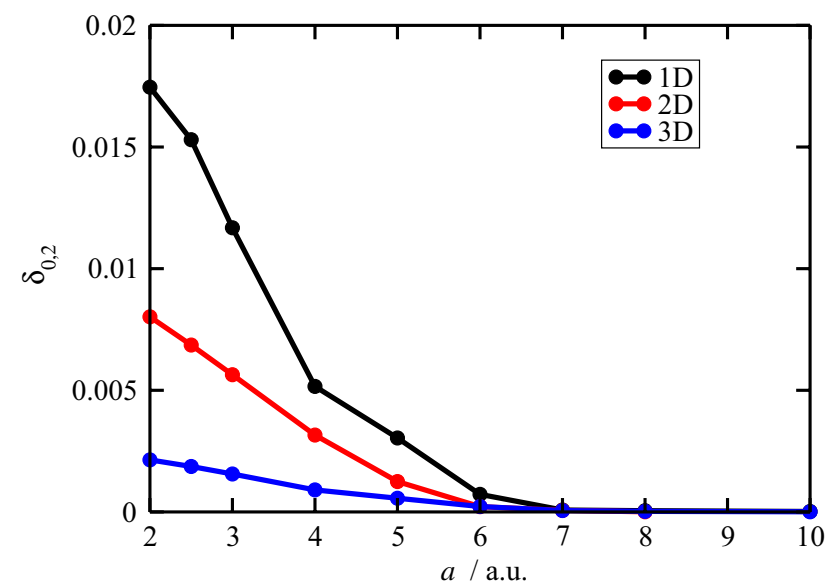

Fig. 5 Dependence of the delocalization index to the neighbor at the distance $2 a$ along the unit cell vector, $\delta_{0,2}$, on the cell parameter $a$ for hydrogen lattices in 1RDMFT calculations

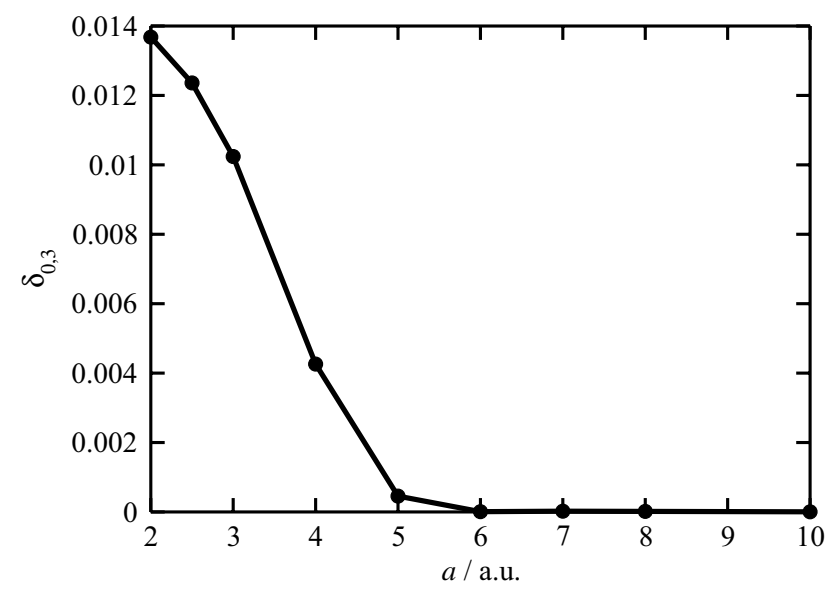

Fig. 6 Dependence of the delocalization index to the neighbor at the distance $3 a$ along the unit cell vector, $\delta_{0,3}$, on the cell parameter $a$ for $1 \mathrm{D}$ hydrogen lattice in $1 \mathrm{RDMFT}$ calculations

In the 1D lattice case, it is also observed that more distant than first neighbor contacts display sigmoidal profiles (Figs. 5, 6). However, this is not the case for 2D and 3D lattices. In these two latter cases, the delocalization indices between QTAIM atoms whose attractors are separated from each other by $2 a$ show profiles with shapes closer to exponential decay, this situation being typical for nonbonding interactions (Fig. 5). The same holds also for contacts between atoms at a distance equal to $3 a$ in the 2D lattice (Fig. 7). At the same time, the DI profiles between next nearest neighbor atoms at a distance equal to $a \sqrt{2}$ in $2 \mathrm{D}$ and 3D lattices (Fig. 8) show profile shapes (Fig. 8) which are difficult to classify unambiguously into the exponential/ sigmoidal categories. Next nearest neighbor profiles for the 3D lattice display even a shallow maximum near the middle

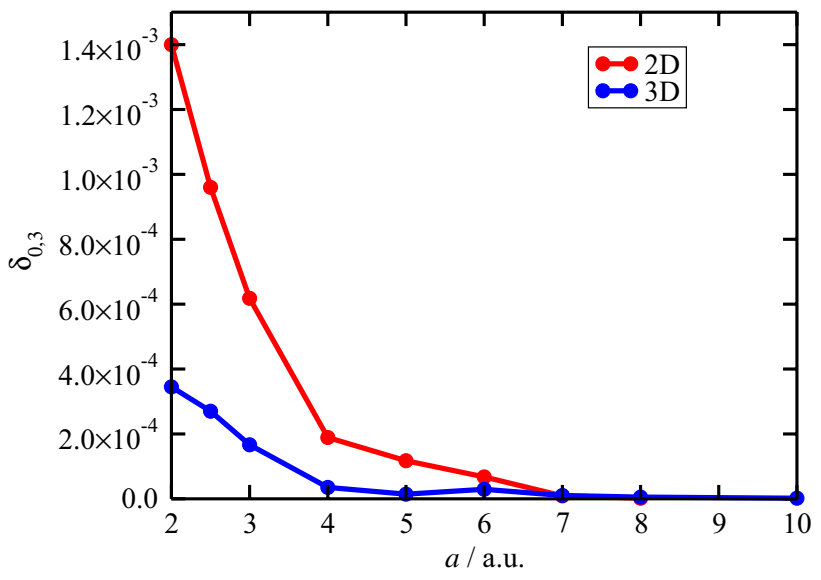

Fig. 7 Dependence of the delocalization index to the neighbor at the distance $3 a$ along the unit cell vector, $\delta_{0,3}$, on the cell parameter $a$ for hydrogen lattices in 1RDMFT calculations

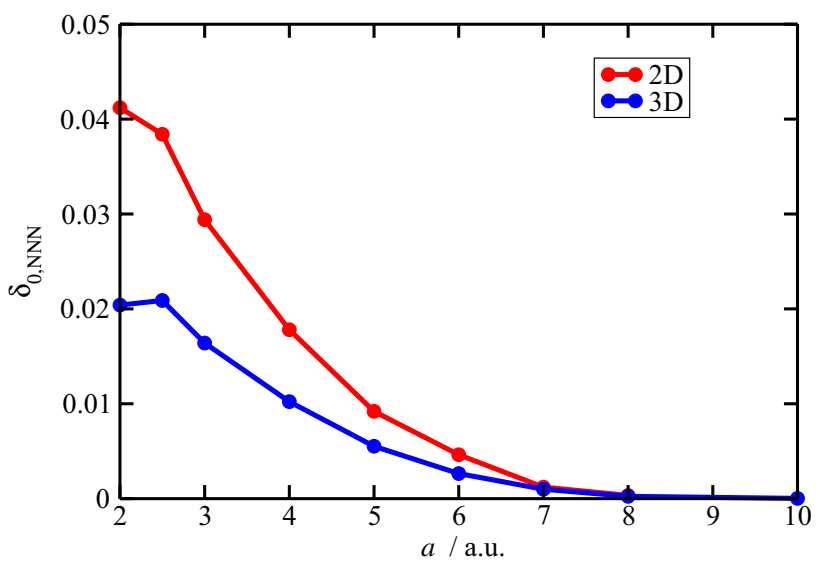

Fig. 8 Dependence of the delocalization index between next nearest QTAIM atoms, $\delta_{0, N N N}$, for 2D and 3D lattices (the distance between attractors is $a \sqrt{2}$ ) on the cell parameter $a$ for hydrogen lattices in 1RDMFT calculations

point of the transition. This provides an interesting insight into the very nature of bonding in metals, showing how different the interactions can be even in the simplest metallic system. The results of this work demonstrate that the chemical bonding between atoms in metals occurs basically among (a few) nearest neighbors, this image delivering a rather localized picture of chemical bonding in contrast to the classical Drude-Sommerfeld model [4] of a delocalized electron gas in which the lattice ions are immersed. Such a locality for the interactions existing in 2D and 3D hydrogen lattices can be rationalized as a consequence of the high coordination numbers in conjunction with the low number of valence electrons that characterizes typical metallic scenarios. The electron sharing of an atom described by its basin-averaged Fermi hole [52-54] provides bonding only 


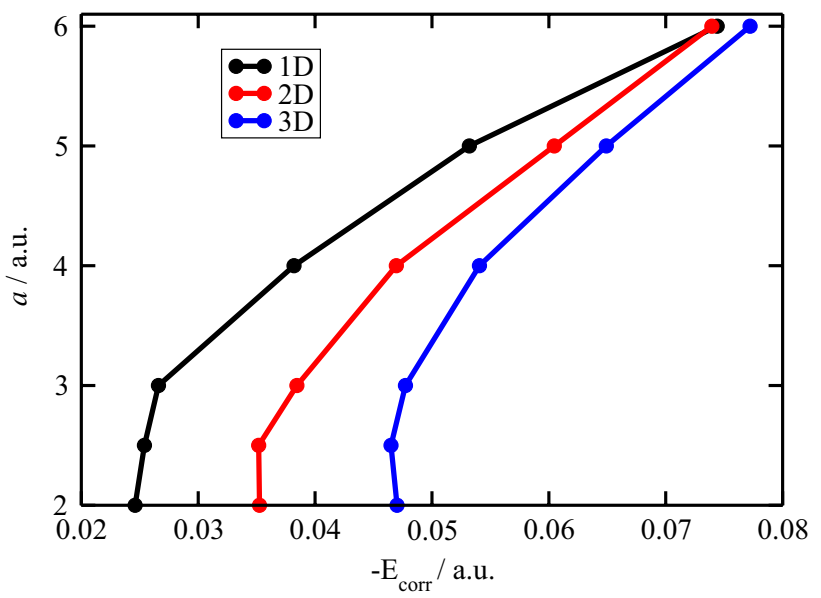

Fig. 9 Relation between the negative 1RDMFT correlation energy (Eq. 5) and the cell parameter $a$ for 1D-3D hydrogen lattices

with a set of relative few neighboring atoms. The extent of this bonding should depend both on the structure defining the coordination number and on the nature of the metal defining the number of available valence electrons.

From the analysis of Hubbard model results, the origin of the sigmoidal profile shapes has been shown [22] to lie in the nonlinear mapping between the interatomic distance and the correlation strength parameter. Figure 9 illustrates the dependence between the 1RDMFT correlation energy calculated using Eq. 5 and the unit cell parameter of the 1D hydrogen lattice, which also shows remarkable nonlinearity. At short distances the correlation energy changes only slowly, whereas at large distances the changes become larger. Quantitatively similar dependence is revealed from DMRG calculations of finite $\mathrm{H}_{50}$ chain [15]. The same nonlinearity is recognized in Fig. 2 by looking at the deviation of the highest $\mathrm{NO}$ occupation from 2, which one can also naively see as a quantity mirroring the strength of the correlation driving the electrons far from each other and preventing double occupation of the same site at large distances. Similar nonlinear dependencies have been recovered for 2D and 3D lattices. The curves in Fig. 9 are shifted to the right in the series 1D-2D-3D, showing that the correlation energy increases with the system dimensionality.

In the case of Hubbard cyclic chains, two types of dependence of the delocalization indices on the correlation strength parameter have been observed previously [22]. The delocalization indices $\delta(0,2 j+1)(j=0,1 \ldots)$ decay monotonically as the correlation strength grows. However, $\delta(0,2 j+2)$ $(j=0,1 \ldots)$ show nonmonotonical dependence: At zero correlation strength they are also equal to zero, then increase as the correlation gets stronger and attain a maximum after which they decay monotonically. From 1RDMFT calculations, we observe only monotonically decaying behavior for all the indices as the correlation strength increases.

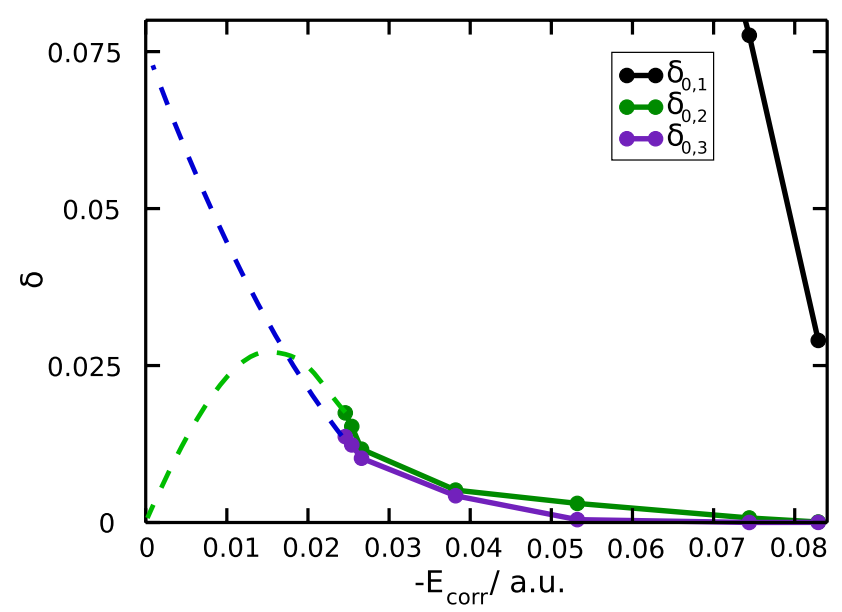

Fig. 10 Relation between first few delocalization indices and the negative 1RDMFT correlation energy for 1D hydrogen lattices. Dots and solid lines connecting them correspond to the calculated results, whereas dashed lines illustrate the anticipated behavior for weak correlation strength

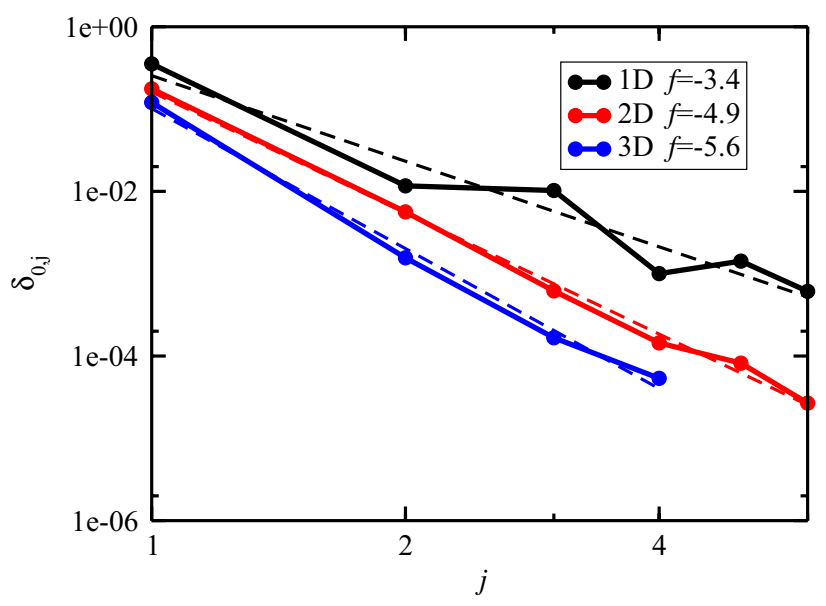

Fig. 11 Spatial dependence of the delocalization indices for 1D-3D lattices with $a=3$ a.u., as obtained from 1RDMFT calculations. Notice the doubly logarithmic scale. Dashed lines show a fit using equation $\delta_{0, j}=j^{-f} \delta_{0,1}$ with the power $f$ specified in the legend

This should mean that in all our calculations the correlation strength is rather strong, in the sense that the weak correlation regime has not been attained (Fig. 10).

Figures 11, 12 and 13 show the spatial decay of the delocalization indices in the 1D-3D lattices with unit cell parameters equal to 3, 5 and 8 a.u., respectively. All the diagrams are plotted in a double logarithmic scale. For small and (to less extent) medium values of the unit cell parameters, (Figs. 11, 12), one observes a linear dependence on these doubly logarithmic plots indicating thus a power law $\delta_{0, j}=j^{-f} \delta_{0,1}$ decay. For $1 \mathrm{D}$ lattice there are visible oscillations for $a=3$ a.u. which have also been observed earlier on 


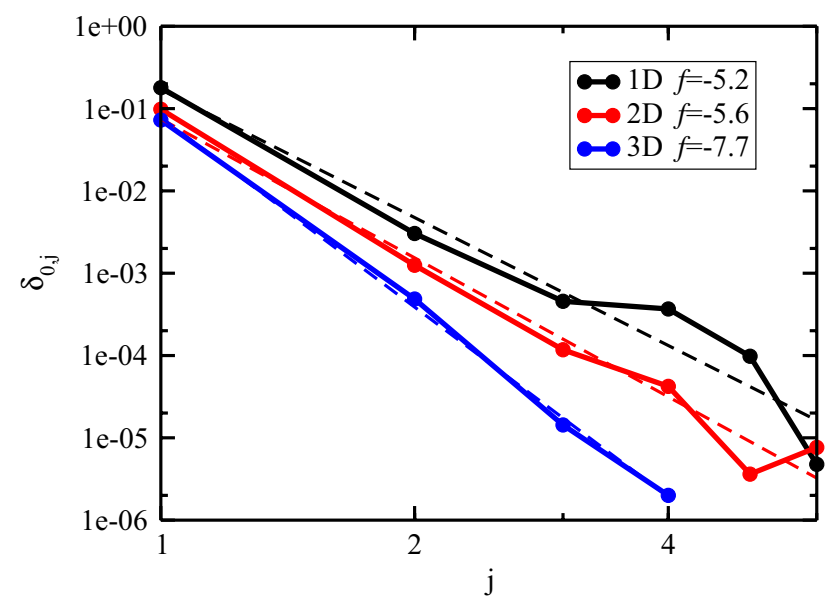

Fig. 12 Spatial dependence of the delocalization indices for 1D-3D lattices with $a=5$ a.u. from 1RDMFT calculations. Notice the doubly logarithmic scale. Dashed lines show a fit using equation $\delta_{0, j}=j^{-f} \delta_{0,1}$ with the power $f$ specified in the legend

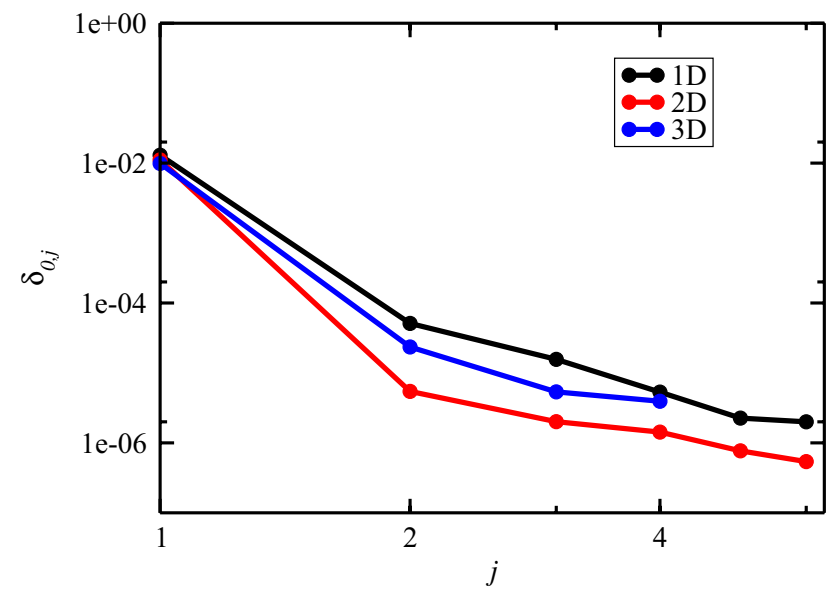

Fig. 13 Spatial dependence of the delocalization indices for 1D-3D lattices with $a=8$ a.u. from 1RDMFT calculations. Notice the doubly logarithmic scale

the basis of DFT calculations [51]. However, in this case the oscillations are clearly weaker than in the DFT results and the situation $\delta_{0, j}<\delta_{0, j+1}$ never shows up. From the analysis of Hubbard models, it was also shown [22] that the oscillations are dumped by electron correlation. In line with that, Fig. 10 indicates that in the 1RDMFT description of the 1D lattice the correlation strength is not very weak and that even at the shortest $a=2$ a.u. parameter these oscillations are dumped. Nevertheless, their presence can be clearly recognized in the plots. For larger unit cell parameters, these oscillations become weaker and starting from $a=5$ a.u. they are not clearly visible anymore (Fig. 12). For 2D and 3D lattices, there are no such pronounced oscillations even for small unit cell parameters, which was also the case for DFT results [51].
The presence of these oscillations is an interesting phenomenon which is related to such different concepts as mesomerism and resonance [55] in chemistry and Friedel oscillations and Ruderman-Kittel-Kasuya-Yosida (RKKY) interactions [4] in solid-state physics. In the last realm, spatial oscillations of the delocalization indices have been shown to be directly related to the Fourier transform of the Fermi surface [56]. Thus, the gradual disappearance of the oscillations during the MT, being dumped by the ever increasing electronic correlation, reflects the progressive disappearance of the Fermi surface under the influence of strong correlations as the metal transforms into the insulator.

An approximate linear dependence (somewhat distorted for large distances probably due to the numerical inaccuracies) holds for the unit cell parameters around $a=6$.a.u. For larger $a$ values, a systematic deviation from the linear dependence appears (Fig. 13) indicating that the power law $\delta_{0, j}=j^{-f} \delta_{0,1}$ is not valid anymore. It is interesting to note that this change occurs at unit cell parameter values close to the middle point of the transition (p. 9).

The power $f$ from the dependence $\delta_{0, j}=j^{-f} \delta_{0,1}$ obtained from 1RDMFT results systematically increases with the dimension of the crystal and with the unit cell parameter. The latter trend has been also observed for molecular ring models calculations of 1D lattices, and it can be directly interrelated to the Fourier transform of the Fermi surface. For instance, for 1D lattices the analytic tight-binding model yields the power $f=2[51,56]$. For 2D system with an isotropic Fermi surface, the power is equal to 3, in compliance with the results of Taraskin [57], whereas for an anisotropic Fermi surface, it can be between 2 to 4 depending on the direction [51]. For a 3D lattice displaying spherical Fermi surface, a fourth-power decay is expected [56].

As in the case of the powers derived from DFT calculations [51], the values obtained here are somewhat higher than the powers derived from simple analytical models, being close to full configuration interaction (FCI) and complete active space (CAS) results for comparable short $\mathrm{H}-\mathrm{H}$ distances. For instance, our value $f=3.45$ for $a=3$ a.u. is very close to $f=3.33$ (CAS) and $f=3.45$ (FCI) for cyclic ring with $(\mathrm{H}-\mathrm{H})=2.7$ a.u. [22]. From our 1 RDMFT calculations for $a=5$ a.u. $f=5.16$ and for $a=6$ a.u. $f=6.85$ which is smaller than CAS and CI values $f=7.54$ and $f=9.09$, which were correspondingly obtained for $a=5.5$ a.u. [22] The larger discrepancies found in this case can probably be attributed to the fact that our fit is obtained for a rather short distance range, whereas the molecular cluster fit was derived from more distant contacts.

Increasing the number of k-points from 16 to 24 and the number of the empty states taken for the NO expansions from 7 to 10 in the case of the 1D lattice does not alter the results appreciably. The same holds if the number of k-point divisions per axis is increased from 8 to 12 in 3D 
lattice. The only remarkable change observed was a very slight increase in electron localization for large values of the unit cell parameter when the number of empty states was increased.

Changing the exponent of the 1RDMFT power function $\lambda$ to 0.5 , this corresponding to moving to Müller's functional does, however, introduce much more noticeable changes in the results. For instance, the energy profile of the transition runs significantly lower, just as it was observed earlier [49], and almost converges to the exact value -0.5 a.u. at large unit cell parameters. The equilibrium shortest interatomic distance lies below 2 a.u., a value which is clearly shorter than the equilibrium distance obtained with other correlated methods. Thus, the Müller functional overbinds the 1D lattice. Surprisingly, this overbinding is not accompanied by electron sharing enhancement as one might expect, but by a marked electron sharing reduction accompanied by a higher electron localization. The localization index evolution profile shifts up, whereas the delocalization index profiles shifts down. Sigmoidal shapes are to a larger extent retained, at least for the first several shortest contacts. Finally, the spatial oscillations of the delocalization indices become less pronounced with Müller's functional than if we use $\lambda=0.656$, this result also being in line with the fact that $\lambda=0.5$ overcorrelates [58].

\section{Conclusions}

1RDMFT calculations employing the power potential with $\lambda=0.656$ have delivered qualitatively correct descriptions of the metal-insulator Mott transitions in 1D-3D hydrogen lattices. Total energies were found to be higher than those coming from other methods. However, their evolution profiles as well as the evolution of the 1RDM eigenvalue spectrum show a correct behavior.

The evolution profiles of localization indices and nearest neighbor delocalization indices for QTAIM atoms in 1D-3D lattices have sigmoidal shapes. Similar shapes have been observed earlier in molecular systems (0D) and 1D Hubbard lattices and have been interpreted as a signature of shared interactions between atoms. At the same time, the profiles for the delocalization indices between more distant neighbors in 2D and 3D lattices show different shapes, having more similarity to the exponential profiles typical in nonbonding interactions. This could mean that, in contrast to the generally accepted conception, bonding interactions in metals can be quite local and might only involve few nearest coordination spheres.

The sigmoidal profile shape for the nearest delocalization indices has been rationalized on the basis of cyclic Hubbard models as a composition of the exponential decay of the delocalization index versus the strength of electronic correlation (which favors electron localization) combined with the nonlinear dependence of the correlation strength versus the unit cell parameter.

Employing 1RDMFT correlation energies, the same types of dependencies have been recovered from 1RDMFT calculations for 1D-3D lattices, confirming this rationale. Another type of dependence for the delocalization indices between next nearest neighbors that was found from Hubbard models, characterized by a maximum at intermediate correlation strengths, has not been observed in the 1RDMFT results here reported. This points at a rather strong correlation strength regime in 1RDMFT calculations, even at the equilibrium distances of the several lattices examined.

The dependence of the delocalization indices with distance in the metallic regime for 1RDMFT calculations was found to roughly follow a power law with $\delta_{0, j}=j^{-f} \delta_{0,1}$, where $j$ is the distance expressed as a multiple of the unit cell parameter. In the $1 \mathrm{D}$ case there are clearly visible oscillations, which are nevertheless much less pronounced than in the DFT results.

The essential damping of these oscillations can be attributed to the significant strength of the electronic correlation as mentioned above. The power $f$ obtained by fitting the reported 1RDMFT results was found to be close to the CAS and FCI results for molecular clusters, at least for short interatomic distances. $f$-values increase with the unit cell parameter as well as with the system dimensionality. In consistence with that, oscillations disappear as the unit cell parameter increases. For large enough values of the unit cell parameters that correspond to the insulating regime, the spatial dependence of the delocalization indices does not obey a power law.

The 1RDMFT calculation results for the 1D lattices reported in this article are consistent with the molecular cluster data. Interestingly, they practically do not depend on the basis set expansion. Changing the power $\lambda$ of the functional has a stronger impact, although the qualitative picture remains unchanged. For 2D and 3D lattices, essentially the same conclusions can be drawn as for 1D lattices.

To conclude, the results of the 1RDMFT calculations here reported confirm the insights into the real-space picture of Mott metal-insulator transitions formulated earlier on the basis of finite molecular calculations and simple analytical models. Armed with this agreement, it has then been possible to generalize them to $2 \mathrm{D}$ and $3 \mathrm{D}$ systems. The results have also showed that even rather simple 1RDMFT functionals can quantitatively correctly describe the spatial evolution of electron localization and sharing for different correlation strengths. We hope that with further developments in this field the real-space analysis of realistic Mott systems based on transition metals will become feasible in the near future. 
Acknowledgements Open access funding provided by Max Planck Society.

Open Access This article is distributed under the terms of the Creative Commons Attribution 4.0 International License (http://creativecommons.org/licenses/by/4.0/), which permits unrestricted use, distribution, and reproduction in any medium, provided you give appropriate credit to the original author(s) and the source, provide a link to the Creative Commons license, and indicate if changes were made.

\section{References}

1. Mott NF (1968) Rev Mod Phys 40:677

2. Gebhard F (1997) The Mott metal-insulator transition. Springer, Berlin

3. Imada M, Fujimori A, Tokura Y (1998) Rev Mod Phys 70:1039

4. Ashcroft NW, Mermin DN (1976) Solid State Phys. Saunders College, Orlando

5. Hubbard J (1963) Proc R Soc Lond 276:238

6. Baeriswyl D, Campbell DK, Carmelo JMP, Guinea F, Louis E (eds) (1995) The Hubbard Model. Springer, US. doi:10.1007/978-1-4899-1042-4

7. Lieb EH, Wu FY (1968) Phys Rev Lett 20:1445

8. Zgid D, Chan GKL (2011) J Chem Phys 134:094115

9. Knizia G, Chan GKL (2012) Phys Rev Lett 109:186404

10. Knizia G, Chan GKL (2013) J Chem Theory Comput 9:1428

11. Stella L, Attaccalite C, Sorella S, Rubio A (2012) Phys Rev Lett 109:186404

12. Kunê̂ J, Lukoyanov AV, Anisimov VI, Scarlettar RT, Pickett WE (2008) Nature Materials 7:198

13. Sharma S, Dewhurst JK, Shallcross S, Gross EKU (2013) Phys Rev Lett 110:116403

14. McWeeny R (1992) Methods of molecular quantum mechanics. Academic Press, London

15. Hachmann J, Cardoen W, Chan GKL (2006) J Chem Phys 125:144101

16. Al-Saidi WA, Zhang S, Krakauer H (2007) J Chem Phys 127:144101

17. Sinitskiy A, Greenman L, Mazziozzi DA (2010) J Chem Phys 133:014104

18. Limacher PA, Ayers PW, Johnson PA, De Baerdemacker S, Van Neck D, Bultinck P (2013) J Chem Theory Comput 9:1394

19. Popelier PLA (2005) Struct Bond 115:1

20. Löwdin PO (1955) Phys Rev 97:1474

21. García-Revilla M, Popelier PLA, Francisco E, Martín Pendás A (2011) J Chem Theory Comput 7:1704

22. Gallo-Bueno A, Kohout M, Martín Pendás A (2016) J Chem Theory Comput 12:3053

23. Gilbert TL (1975) Phys Rev B12:2111

24. Ambrosch-Draxl C (2004) Phys Scr T109:48
25. Bader RFW, Stephens ME (1974) Chem Phys Lett 25:445

26. Bader RFW, Stephens ME (1975) J Am Chem Soc 97:7391

27. Sharma S, Dewhurs JK, Lathiotakis NN, Gross EK (2008) Phys Rev B 78:201103

28. Coleman AJ (1963) Rev Mod Phys 35:668

29. Müller AMK (1984) Phys Lett A 105:446

30. Piris M (2007) In: Mazziotti D (ed) Reduced-density-matrixmechanics: with applications to many-electron atoms and molecules, a special volume of advances in chemical physics, vol 134. Wiley, Hoboken

31. Marques MLA, Lathiotakis NN (2008) Phys Rev A 77:032509

32. Piris M, Ugalde JM (2014) Int J Quant Chem 114:1169

33. Cioslowski J, Pernal K (1999) J Chem Phys 111:3396

34. Cioslowski J, Piris M, Matito E (2015) J Chem Phys 143:214101

35. Program Elk versions 3.1.12. (2015) http://elk.sourceforge.net. Accessed Dec 2016

36. Perdew JP, Wang Y (1992) Phys Rev B 45:13244

37. Singh DJ (1994) Planewaves, pseudopotentials and the LAPW method. Kluwer Academic, Boston

38. Kutzelnigg W (2002) In: Rychlewski J (ed) Explicitly correlated wave functions chemistry and physics: theory and applications. Kluwer, The Netherlands, pp 14-17

39. Bader RFW (1990) Atoms in molecules: a quantum theory. Oxford University Press, Oxford

40. Fradera X, Austen MA, Bader RFW (1999) J Phys Chem A 103:304

41. Mayer I (1983) Chem Phys Lett 97:270

42. Jug K, Fasold E, Gopinathan MS (1989) J Comput Chem 10:965

43. Ángyán J, Loos M, Mayer I (1994) J Phys Chem 98:5244

44. Buijse MA, Baerends EJ (2002) Mol Phys 100:401

45. Fulton RL (1993) J Phys Chem 97:7516

46. Kohout M (2016) Program DGrid-4.7. Dresden

47. Baranov AI, Kohout M (2011) J Comput Chem 32:2064

48. Ziesche P (2000) J Mol Str (Theochem) 527:35

49. Lathiotakis NN, Sharma S, Dewhurst JK, Eich FG, Marques MAL, Gross EKU (2009) Phys Rev A 79:040501

50. Baranov AI, Kohout M (2011) Acta Cryst A 67:C115

51. Gallo-Bueno A, Francisco E, Martin Pendás A (2016) Phys Chem Chem Phys 18:11772

52. Ponec R (1997) J Math Chem 21:323

53. Ponec R (1998) J Math Chem 23:85

54. Baranov AI, Ponec R, Kohout M (2012) J Chem Phys 137:214109

55. Pauling L (1960) The nature of chemical bond, 3rd edn. Cornell University Press, Ithaca

56. Baranov AI, Ponec R, Kohout M (2016) In: Joswig JO, Springborg M (eds) Chemical modelling. Royal Society of Chemistry, Cambridge

57. Taraskin SN, Drabold DA, Elliot SR (2002) Phys Rev B 66:233101

58. Lathiotakis NN, Marques MAL (2008) J Chem Phys 128:184103 